\title{
A 10-YEAR PROSPECTIVE STUDY OF NOISE AND HEARING
} IN CONSTRUCTION WORKERS

Noah Seixas, ${ }^{1}$ Rick Neitzel, ${ }^{1}$ Lianne Sheppard, ${ }^{1}$ Bert Stover, ${ }^{1}$ David Mills, ${ }^{1}$ Pat Feeney, Sharon Kujawa² 'University of Washington, Seattle, USA; ${ }^{2}$ Massachusetts Eye and Ear Hospital, Boston, USA

10.1136/oemed-2011-100382.136

Objectives To quantitatively evaluate the effect of noise exposure on hearing, an inception cohort of construction workers were monitored over a 10 year period.

Methods Construction apprentices and student controls $(\mathrm{n}=316)$ were enrolled in 1999 and assessed for hearing approximately annually through 2009 . At each of 2896 time points, hearing was measured using pure-tone audiometry, and health, work history, non-occupational exposures and other information was collected via survey. 1310 full shift noise measurements were used to estimate task-based individual average Leq noise exposures. Changes in measurement techniques necessitated analyses within two phases, as well as over the whole study period.

Results In unadjusted analyses, adverse changes over time and in relation to exposure were observed in hearing thresholds at 3 and $4 \mathrm{kHz}$. At $6 \mathrm{kHz}$ there was an apparent improvement in hearing. Changes in thresholds within each phase were consistent with the overall trend. The effect at $4 \mathrm{kHz}$ demonstrated a loss of $0.81(+0.11) \mathrm{dB}$ per year among construction workers, compared to $0.34(+0.09) \mathrm{dB}$ among controls. Linear mixed models were developed to estimate the change in hearing in relation to exposure, while controlling for ear within subject, and relevant covariates, and demonstrate similar effects.

Conclusions Hearing thresholds at noise-sensitive frequencies increased with exposure over a 10 year study period, consistent with the predicted loss for an $85 \mathrm{dBA}$ exposure. However, challenges of monitoring construction workers over this extended period of time make the magnitude of the effect uncertain. 